\title{
Probing upper thermospheric neutral densities at Mars using electron reflectometry
}

\author{
R. J. Lillis, ${ }^{1,2}$ J. H. Engel, ${ }^{1,2}$ D. L. Mitchell, ${ }^{1}$ D. A. Brain, ${ }^{1}$ R. P. Lin, ${ }^{1,2}$ S.W. Bougher, ${ }^{3}$ \\ and M. H. Acuña ${ }^{4}$ \\ Received 8 August 2005; revised 1 November 2005; accepted 9 November 2005; published 15 December 2005.
}

[1] We present a new technique for inferring neutral densities in the Martian upper atmosphere from atmospheric absorption of magnetically reflected solar wind electrons. Using electron loss cone measurements from the Magnetometer/Electron Reflectometer (MAG/ER) experiment on board Mars Global Surveyor (MGS), we derive upper thermospheric $(\sim 160-230 \mathrm{~km}$ altitude) densities in the southern hemisphere from $160^{\circ}$ to $200^{\circ} \mathrm{E}$ at 2am local time, continuously from 1999 to 2005 . We find a mean density of $0.027 \mathrm{~kg} / \mathrm{km}^{3}$ at $160 \mathrm{~km}$ and observe a latitude-dependent, repeatable seasonal variation of a factor of 1.8-4, with inter-annual differences and consistently lower winter densities at $50^{\circ}-55^{\circ} \mathrm{S}$, compared with $0^{\circ}-30^{\circ} \mathrm{S}$. The mean densities, overall seasonal variation and latitude dependence are in general agreement with the Mars Thermosphere Global Circulation Model (MTGCM). These measurements are important for improved understanding of the dynamics of Mars' upper atmosphere and for planning spacecraft aerobraking maneuvers. Citation: Lillis, R. J., J. H. Engel, D. L. Mitchell, D. A. Brain, R. P. Lin, S. W. Bougher, and M. H. Acuña (2005), Probing upper thermospheric neutral densities at Mars using electron reflectometry, Geophys. Res. Lett., 32, L23204, doi:10.1029/2005GL024337.

\section{Introduction}

[2] The Martian thermosphere (above $110 \mathrm{~km}$ ) has been sampled by several spacecraft. The Viking 1,2 landers (below $200 \mathrm{~km}$ ) and Pathfinder lander (below $161 \mathrm{~km}$ ) each yielded one vertical profile under solar minimum EUV flux conditions [Nier and McElroy, 1977; Magalhães, 1999]. MGS accelerometer data have provided over 1600 vertical profiles of atmospheric density from $110 \mathrm{~km}$ to $170 \mathrm{~km}$ [Keating et al., 1999] in solar minimum to medium conditions. X-band Doppler tracking of MGS during the Science Phasing Orbits (SPO) gave $\sim 200$ density measurements between 172 and $178 \mathrm{~km}$ over a 6-month period in 1998 under solar medium conditions [Tracadas et al., 2001]. Finally, Mars Odyssey accelerometers gave 600 vertical profiles from 95 to $170 \mathrm{~km}$ during solar maximum in late 2001 [Keating et al., 2003]. While these measurements have been adequate to partially validate models of

\footnotetext{
${ }^{1}$ Space Sciences Laboratory, University of California, Berkeley, California, USA.

${ }^{2}$ Department of Physics, University of California, Berkeley, California, USA.

${ }^{3}$ Atmospheric, Oceanic and Space Sciences, University of Michigan, Ann Arbor, Michigan, USA.

${ }^{4}$ NASA Goddard Space Flight Center, Greenbelt, Maryland, USA.
}

Copyright 2005 by the American Geophysical Union. 0094-8276/05/2005GL024337 thermospheric structure, such as the Mars Thermosphere General Circulation Model (MTGCM) [Bougher et al., 2000; S. Bougher et al., Polar warming in the Mars lower thermosphere: Seasonal variations owing to changing insolation and dust distributions, submitted to Geophysical Research Letters, 2005], the sampling is not nearly complete with respect to Mars' seasonal longitude $\left(\mathrm{L}_{\mathrm{s}}\right)$, solar activity, latitude and local time.

[3] The magnetic mirror effect, that is, the reflection of charged particles from regions of increased magnetic field strength, has been successfully used to map crustal magnetic fields on the Moon [Lin, 1979; Halekas et al., 2001]. The MGS Electron Reflectometer (ER) was designed to use this effect to remotely probe crustal magnetic fields on Mars [Acuña et al., 1992]. To apply this technique in practice, Lillis et al [2004] performed the necessary modeling of the interaction of solar wind electrons with the significant neutral atmosphere, whose density profile determines the pitch angle-dependent attenuation of electron flux. Such a density profile must be assumed a priori to solve for the crustal magnetic field strength at specific altitudes. This is important for the regions of weak crustal magnetic field where the magnetic field measurements at spacecraft $(\mathrm{S} / \mathrm{C})$ altitude cannot uniquely resolve crustal fields from fields induced by the solar wind interaction with Mars' ionosphere in a single orbital pass [Acuña et al., 2001]. However, in regions where the crustal magnetic field is strong, the induced field is negligible by comparison. The field geometry there can be well-approximated by spherical harmonic expansions [Cain et al., 2003] and the atmospheric neutral density profile can be obtained [Lillis et al., 2003]. We describe this method for inferring atmospheric neutral densities, using atmospheric absorption of magnetically reflected solar wind electrons, and present results from 160 to $230 \mathrm{~km}$ in the southern hemisphere at $2 \mathrm{am}$ local time over the entire mapping orbit phase of MGS from May 1999 to February 2005. Though the ER was not designed for this purpose and the uncertainties are large for a single measurement, we will show that long-term trends in densities can be extracted through averaging over timescales of several weeks.

\section{Modeling Electron Loss Cones}

[4] Solar wind electrons on open magnetic field lines (those connected to both the planetary crust and the interplanetary magnetic field) follow a spiral path and experience an average force opposite in direction to the gradient of the local magnetic field. Thus, the converging field lines in the vicinity of crustal magnetic anomalies act as magnetic 'mirrors' for these electrons. An electron with an initial 


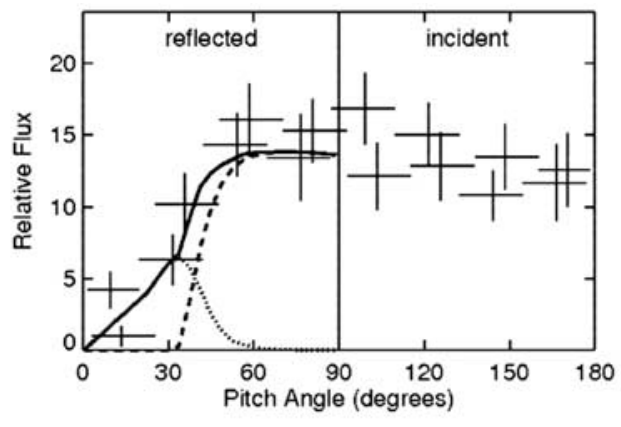

Figure 1. Example MAG/ER 190-eV dimensionless electron flux in 16 pitch angle bins. Bins overlap because each pitch angle is sampled by 2 sides of the $360^{\circ}$ instrument anode [Mitchell et al., 2001]. Horizontal bars give bin widths, while vertical error bars include both statistical and calibration $(<5 \%)$ uncertainties. A best-fit Monte Carlo kinetic model (solid black curve) is the sum of magnetically reflected (dashed) and backscattered (dotted) electrons [see Lillis et al., 2004, section 2].

incident pitch angle $\alpha$ at the $\mathrm{S} / \mathrm{C}$ will reflect back to the $\mathrm{S} / \mathrm{C}$ with a pitch angle of $180^{\circ}-\alpha$. The reflection point along the field line, $x_{r}$, occurs where $\left|B\left(x_{r}\right)\right|=\left|B_{S C}\right| / \sin ^{2} \alpha$, where $\left|B\left(x_{r}\right)\right|$ and $\left|B_{S C}\right|$ are the magnetic field magnitudes at $x_{r}$ and the S/C respectively [Parks, 2004]. The strength of the magnetic field increases with decreasing distance to the crustal source. Thus, electrons with pitch angles near 90 degrees at the $\mathrm{S} / \mathrm{C}$ reflect above most of the atmosphere and are not attenuated, while electrons with pitch angles closer to $0^{\circ}$ (or $180^{\circ}$ ) at the spacecraft reflect at lower altitudes and so are more likely to be absorbed by the atmosphere before reflecting. Therefore, when MGS is on an open field line, the ER sees a pitch angle-dependent attenuation of electron flux, known as a loss cone (Figure 1).

[5] We model the survival probability of an electron following a spiral path in an inhomogeneous magnetic field while passing through a given atmospheric density profile. The probability $P_{\text {surv }}$ of an electron not being scattered after traveling a distance $x$ along a field line can be shown to be [from Lillis et al., 2004, equation (2)]:

$$
P_{\text {surv }}(x)=\exp \left[-\sum_{i} \sigma_{i} \int_{0}^{x} \frac{n_{i}\left(x^{\prime}\right)}{\sqrt{1-\frac{B\left(x^{\prime}\right)}{B_{0}} \sin ^{2} \alpha_{0}}} d x^{\prime}\right]
$$

where $\Sigma$ is the sum over the major upper thermospheric neutral species; $\sigma_{\iota}$ are the total energy-dependent electronneutral scattering cross-sections, $n_{i}\left(x^{\prime}\right)$ are the number densities of the neutral species at $x=x^{\prime}, B_{0}$ and $B\left(x^{\prime}\right)$ are the magnitudes of the magnetic field at $x=0, x^{\prime}$ and $\alpha_{0}$ is the pitch-angle of the electron at $x=0$. If all scattered electrons are assumed lost, this equation predicts a simple loss cone (Figure 1, dashed line). However, Monte Carlomodeling of the scattering of individual electrons is required to account for the backscattered electron population (Figure 1, dotted line), which must be included to describe accurately the shape of loss cones observed by MAG/ER [see Lillis et al., 2004, section 2]. Figure 2 illustrates the relationship between $P_{\text {surv }}$, pitch angle, and reflection altitude for two extrema MTGCM model atmospheres, showing the existence of an effective range of altitudes (approx. 160-230 km) over which the loss cone is formed. It can be shown that $P_{\text {surv }}$ is most sensitive to column depth of atmosphere along the electron's spiral path at a value of $1 / e$. This corresponds to $\sim 160 \mathrm{~km}$, which we choose as our reference altitude.

\section{Inferring Atmospheric Densities}

[6] The two main constituents in the Martian upper thermosphere are $\mathrm{CO}_{2}$ and atomic oxygen [Nier and McElroy, 1977]. Above the exobase, the two species are not mixed and so have different scale heights based upon their relative molecular masses (44 and 16 respectively) and temperatures. We parameterize a 2 -species isothermal atmosphere, expressing density as a function of height as:

$$
n_{i}(h)=n_{0 i} \exp \left[-\int_{h_{0}}^{h} \frac{d h^{\prime}}{H_{i}\left(h^{\prime}\right)}\right], H_{i}(h)=\frac{k T\left(R_{\text {Mars }}+h\right)^{2}}{m_{i} G M_{\text {Mars }}}
$$

where $h_{0}$ is a reference altitude (160 km, see section 2), $i$ is the species index, $n_{0 i}$ is the number density at $h_{0}, H_{i}(h)$ is the altitude-dependent scale height, $T$ is the constant temperature above $h_{0}, k$ is the Boltzmann constant, $G$ is the gravitational constant, $m_{i}$ is the mass of a molecule of species $i$ and $R_{\text {Mars }}, M_{\text {Mars }}$ are the radius and mass of Mars respectively. $n_{0-\mathrm{CO} 2},, n_{0-\mathrm{O} 1}$ and $T$ are the free parameters which we may adjust to fit the observed loss cone.

[7] To use equations (1), (2) to fit for the densities and temperatures of the neutral atmosphere, the magnetic field magnitude must be specified as a function of distance along the field line, denoted $B(x)$ in equation (1). Cain et al. [2003] have modeled the magnetic field on the Martian nightside as an expansion in spherical harmonics to degree and order 90, hereby referred to as ' $B_{F S U}$ ' (from Florida State University). In regions of Mars where the crustal magnetic field is weaker than or comparable to the field induced by the interaction between the solar wind and Mars'

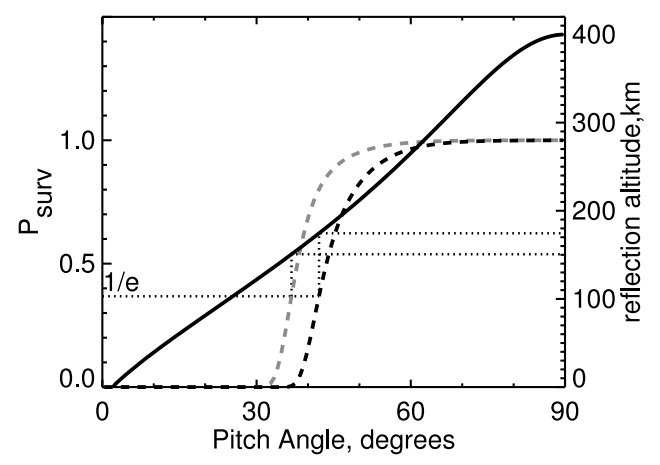

Figure 2. $190 \mathrm{eV}$ electron reflection altitude (solid black line, right y-axis) and survival probability (gray and black dashed lines, left $y$-axis) as a function of pitch angle at the $\mathrm{S} / \mathrm{C}$ (x-axis) are plotted for a typical crustal magnetic field profile $B(x)\left(\right.$ at $\left.6^{\circ} \mathrm{S}, 39^{\circ} \mathrm{E}\right)$ and for 2 possible extreme MTGCM night side atmospheres (solar min, aphelion: grey dashed lines; solar max, perihelion: black dashed lines). Dotted lines show how to relate $P_{\text {surv }}$ to a pitch angle and how that pitch angle corresponds to a magnetic reflection altitude, using the value for $P_{\text {surv }}$ of maximum sensitivity $(1 / \mathrm{e})$. 


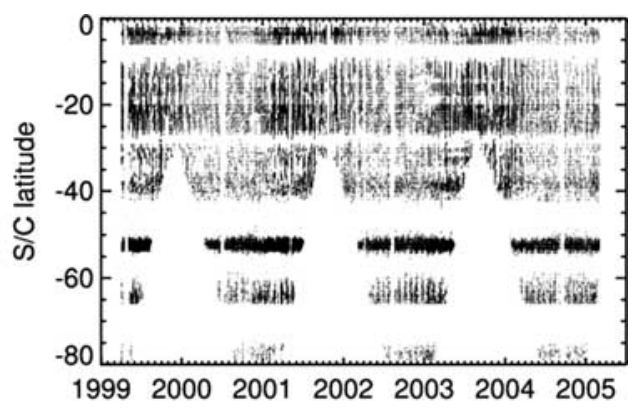

Figure 3. Data coverage versus year and latitude used in this study. Variable data density is due to variable spacecraft telemetry rates and photo-electron contamination of loss cones close to the south pole in southern summer.

conducting ionosphere [Brain et al., 2003], the magnetic field profile $B(x)$ can be highly variable and crustal field models are unreliable. However, where the crustal field is strong compared to the induced field, $B_{F S U}$ reproduces magnetometer measurements with acceptable accuracy [Cain et al., 2003]. Therefore, we limit this study to regions where the absolute value of the radial component of the magnetic field (characterizing best the crustal field [Connerney et al., 2001]) at $400 \mathrm{~km}$ is greater than $50 \mathrm{nT}$ and thus $B_{F S U}$ can be trusted. We require that the measured field at the $\mathrm{S} / \mathrm{C}$ position must agree with the FSU model to within 5\% in magnitude and $10^{\circ}$ in direction. The resulting coverage is $\sim 2 \%$ of the planet, in the southern hemisphere, between $160^{\circ}$ and $200^{\circ} \mathrm{E}$ longitude and is seasonally dependent because we only examine data when the $\mathrm{S} / \mathrm{C}$ is in shadow (solar zenith angle $>120^{\circ}$ ) to avoid photo-electron contamination of the loss cones (see Figure 3). While topographic effects on densities dissipate strongly by $160 \mathrm{~km}$ in day time [Withers et al., 2003, Figure 6], the night side case is uncertain at present, so there may be a geographic selection bias in our inferred densities. Due to the large mass difference between electrons and neutrals, the electron precipitation itself causes no significant heating of neutrals [Rees and Fuller-Rowell, 1991].

[8] We calculated loss cone shapes (using equations (1), (2) and convolving to instrumental pitch angle bins) for each of $\sim 1600$ appropriate $1^{\circ} \times 1^{\circ}$ geographic pixels, in the $116,191,313 \mathrm{eV}$ channels, for a range of 6300 possible atmospheres, parameterized by all combinations of 30 logarithmically-spaced values of $n_{0-C O 2},\left(5 \times 10^{6}-5 \times\right.$ $\left.10^{12} \mathrm{~cm}^{-3}\right), 30$ values of $n_{0-O 1}\left(5 \times 10^{6}-5 \times 10^{12} \mathrm{~cm}^{-3}\right)$ and 7 values of $T(140-200 \mathrm{~K})$. These bounding values were chosen to bracket the physically reasonable predictions of the MTGCM.

[9] We compare each loss cone in a measured pitch-angle distribution to the prepared loss cones and calculate the goodness-of-fit $\chi^{2}$ for every combination of $n_{0-\mathrm{CO} 2}, n_{0-\mathrm{O} 1}, T$ in our search grid and find its minimum. This point in parameter space is our best fit and corresponds to an isothermal density profile above $160 \mathrm{~km}$. The uncertainty in this density at a given altitude is quantified by considering all points within the 1- $\sigma$ error hyper-ellipsoid around the minimum of $\chi^{2}$ and assigning upper and lower bound mass density values to be the highest/lowest mass density at that altitude as calculated using the values of $n_{0-\mathrm{CO} 2}, n_{0-\mathrm{O} 1}$, $T$ in that ellipsoid in equation (2). Due to instrumental uncertainties in the loss cone shape, $n_{0-\mathrm{CO} 2}, n_{0-\mathrm{O} 1}$ are almost entirely non-orthogonal parameters: only their linear combination into mass density is constrained, thus we examine only mass density. Best-fit temperatures average $185-195 \mathrm{~K}$ though the error ellipsoid spans the entire constrained range of $T$ in about 2/3 of cases. However, the contribution of errors in $n_{0-\mathrm{CO} 2}$ to the uncertainty in inferred mass densities decreases with altitude $\left(\mathrm{CO}_{2}\right.$ has a smaller scale height). Thus, total errors don't increase with altitude (see Figure 4b). These errors can be considerable for a single measurement, usually a factor of 3 to 20 . We look for long-term changes by averaging over hundreds of measurements in 2-month intervals. We take our error to be the mean of all calculated uncertainties in an interval divided by the root of the number of measurements. Within our 5\% magnetic field error envelope, there is an annual expansion/compression of night side crustal fields due to their seasonally changing solar zenith angle (SZA). This causes a seasonal bias in derived densities of $40 \%$ (equatorial) to $70 \%$ (at $55^{\circ} \mathrm{S}$ ). In addition, absolute uncertainties in $B_{F S U}$ at lower altitudes contribute non-seasonally-dependent systematic errors of up to $50 \%$.

\section{Results and Discussion}

[10] Figure 4a plots latitude-binned and latitude-summed 2-month logarithmic averages of mass densities at $160 \mathrm{~km}$, collected from $160^{\circ}-200^{\circ} \mathrm{E}$. We find a mean density of $0.027 \mathrm{~kg} / \mathrm{km}^{3}$ at $160 \mathrm{~km}$ at 2 a.m. and an overall seasonal
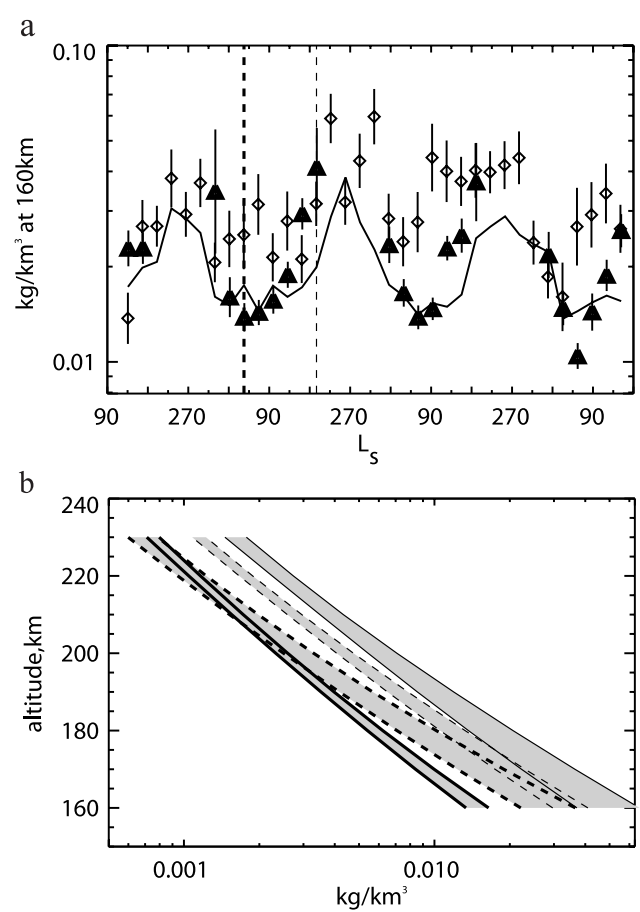

Figure 4. 2-month logarithmically-averaged mass densities collected from $160^{\circ}-200^{\circ} \mathrm{E}$. (a) density at $160 \mathrm{~km}$ from $0^{\circ}-30^{\circ} \mathrm{S}$ (diamonds), $50^{\circ}-55^{\circ} \mathrm{S}$ (triangles) with RMS uncertainties, and all latitudes (solid line), versus Mars $L_{s}$, from 1999 to 2005. Aphelion, perihelion occur at $\mathrm{L}_{\mathrm{s}}=$ $70.4^{\circ}, 250.1^{\circ}$. (b) altitude-density profiles with error envelopes (shaded) for two 2-month intervals indicated by thick and thin vertical dashed lines in a). Thick lines represent $\mathrm{L}_{\mathrm{s}}=17^{\circ}-49^{\circ}$, thin lines $\mathrm{L}_{\mathrm{s}}=178^{\circ}-210^{\circ}$, dashed lines a latitude range of $0^{\circ}-30^{\circ} \mathrm{S}$ and solid lines $50^{\circ}-55^{\circ} \mathrm{S}$. 
expansion/contraction of the thermosphere (Figure 4a, solid line), in general agreement with MTGCM predictions averaged over the same longitude range. Within this pattern, however, there are latitudinal and inter-annual differences. Densities at mid-latitude $\left(50^{\circ}-55^{\circ} \mathrm{S}\right)$, where the crustal magnetic field is strongest, show a consistent seasonal variation of a factor of 3-4, with the lowest densities $\left(0.01 \mathrm{~kg} / \mathrm{km}^{3}\right)$ occurring in the final Martian year of the study. At equatorial latitudes $\left(0^{\circ}-30^{\circ} \mathrm{S}\right)$, where errors are larger, the variation appears weaker: a factor of 1.7-2.8.

[11] The latitudinal difference is greatest near aphelion $\left(\mathrm{L}_{\mathrm{s}}=70^{\circ}\right)$, with a decrease in density from the equatorial to mid-latitude band of a factor of 1.5-3 while the MTGCM predicts a factor of 3.5. This may result from a tidal phasing offset and/or too-strong tidal amplitudes in the night side Mars thermosphere as simulated within the MTGCM during aphelion conditions. Densities in late spring and early autumn are almost indistinguishable between the bands. Figure $4 \mathrm{~b}$ compares altitude-density profiles for four sample 2-month intervals: equatorial and mid-latitudes for $\mathrm{L}_{\mathrm{s}}=$ $17^{\circ}-49^{\circ}$ and $178^{\circ}-210^{\circ}$, showing the aforementioned, and pattern to be consistent pattern at all altitudes probed. Derived mass density scale heights range from 18 to $23 \mathrm{~km}$ and appear to be slightly lower at the equator than at mid-latitude.

[12] Three separate checks were performed on the dataset of $\sim 11,000$ measurements to ensure the seasonal variation was real: a least-squares sinusoidal fit to the logarithms of all densities at each altitude, giving a periods of $0.97 \pm 0.03$ Martian years; periodograms [Thong et al., 2004] of the densities, showing very strong peaks in Lomb-normalized power spectral density $(>100)$ at a period of $\sim 1$ Martian year (corresponding to negligible probabilities, $<10^{-42}$, of being due to random noise in the data); and KolmogorovSmirnov tests, which reveal probabilities of $<10^{-12}$ that latitude-summed winter and summer densities could be drawn from the same parent distribution and $<10^{-5}$ that winter densities from the two latitude bands could be drawn from the same distribution.

[13] This technique can be applied anywhere solar wind electrons precipitate along open field lines onto a planetary atmosphere in a region of a significant, known magnetic gradient. Further, electron reflection instrumentation with better pitch-angle resolution, energy range and higher sensitivity could, in principle, provide neutral density profiles from $\sim 130 \mathrm{~km}$ to $\sim 300 \mathrm{~km}$ with high time resolution.

[14] Acknowledgment. This work was supported by NASA grants JPL $1271721-05 / 06$ and JPL 08668-01/07.

\section{References}

Acuña, M. H., et al. (1992), The Mars Observer magnetic fields investigation, J. Geophys. Res., 97, 7799.

Acuña, M. H., et al. (2001), Magnetic field of Mars: Summary of results from the aerobraking and mapping orbits, J. Geophys. Res., $106,23,403$.
Bougher, S. W., S. Engle, R. G. Roble, and B. Foster (2000), Comparative terrestrial planet thermospheres: 3 . Solar cycle variation of global structure and winds at solstices, J. Geophys. Res., 105, 3091.

Brain, D. A., F. Bagenal, M. H. Acuña, and J. E. P. Connerney (2003), Martian magnetic morphology: Contributions from the solar wind and crust, J. Geophys. Res., 108(A12), 1424, doi:10.1029/2002JA009482.

Cain, J. C., B. B. Ferguson, and D. Mozzoni (2003), An $n=90$ internal potential function of the Martian crustal magnetic field, J. Geophys. Res., 108(E2), 5008, doi:10.1029/2000JE001487.

Connerney, J. E. P., M. H. Acuña, P. Wasilewski, G. Kletetschka, N. F. Ness, H. Reme, R. P. Lin, and D. Mitchell (2001), The global magnetic field of Mars and implications for crustal evolution, Geophys. Res. Lett., 28,4015 .

Halekas, J., D. L. Mitchell, R. P. Lin, S. Frey, L. L. Hood, M. H. Acuña, and A. B. Binder (2001), Mapping of crustal magnetic anomalies on the lunar near side by the Lunar Prospector Magnetometer/Electron Reflectometer, J. Geophys. Res., 106, 27,841.

Keating, G. M., R. H. Tolson, S. N. Noll, T. J. Schellenberg, R. L. Stephens, M. Bradford, S. W. Bougher, and J. L. Hollingsworth (1999), First global mapping of the Mars thermosphere, paper presented at 31st Annual Meeting of the Division for Planetary Sciences, Am. Astron. Soc., Padua, Italy.

Keating, G. M., M. Theriot, R. Tolson, S. Bougher, F. Forget, and J. Forbes (2003), Global measurements of the Mars upper atmosphere: In situ accelerometer measurements from Mars Odyssey 2001 and Mars Global Surveyor, Lunar Planet. Sci., XXXIV, abstract 1142.

Lillis, R. J., D. L. Mitchell, R. P. Lin, and M. H. Acuña (2003), Measuring Mars' atmospheric neutral density from 160 to $320 \mathrm{~km}$ altitude with the MGS Electron Reflectometer, Eos Trans. AGU, 84(46), Fall Meet. Suppl., abstract P52B-05.

Lillis, R. J., D. L. Mitchell, R. P. Lin, J. E. P. Connerney, and M. H. Acuña (2004), Mapping crustal magnetic fields at Mars using electron reflectometry, Geophys. Res. Lett., 31, L15702, doi:10.1029/2004GL020189.

Lin, R. P. (1979), High spatial resolution measurements of surface magnetic fields of the lunar frontside, Proc. Lunar Planet. Sci. Conf. 10th, 2259.

Magalhães, J. A. (1999), Results from the Mars Pathfinder atmospheric structure investigation, J. Geophys. Res., 104, 8943.

Mitchell, D. L., R. P. Lin, C. Mazelle, H. Rème, P. A. Cloutier, J. E. P. Connerney, M. H. Acuña, and N. F. Ness (2001), Probing Mars' crustal magnetic field and ionosphere with the MGS Electron Reflectometer, J. Geophys. Res., 106, 23,419.

Nier, A. O., and M. B. McElroy (1977), Composition and structure of Mars' upper atmosphere: Results from the neutral mass spectrometers on Viking 1 and 2, J. Geophys. Res., 82, 4341.

Parks, G. K. (2004), Physics of Space Plasmas, 2nd ed., Westview, Boulder, Colo.

Rees, D., and T. J. Fuller-Rowell (1991), Thermospheric response and feedback to auroral inputs, in Auroral Physics, edited by C.-I. Meng, M. J. Rycroft, and L. A. Frank, pp. 51-65, Cambridge Univ. Press, New York.

Thong, T., J. McNames, M. Aboy, and B. S. Oken (2004), Averaged Lomb periodograms for non-uniform sampling, paper presented at 17th Biennial International EURASIP Conference: Biosignal 2004, Eur. Assoc. for Signal, Speech and Image Process., Brno, Czech Republic, 23-25 June.

Tracadas, P. W., M. Zuber, D. Smith, and F. G. Lemoine (2001), Density structure of the upper thermosphere of Mars from measurements of air drag on the Mars Global Surveyor spacecraft, J. Geophys. Res., 106, 23,349.

Withers, P., S. W. Bougher, and G. M. Keating (2003), The effects of topographically controlled thermal tides in the Martian upper atmosphere as seen by the MGS accelerometer, Icarus, 164, 14.

M. H. Acuña, NASA Goddard Space Flight Center, Greenbelt, MD 20771, USA.

S. W. Bougher, Atmospheric, Oceanic and Space Sciences, University of Michigan, Ann Arbor, MI 48109-2143, USA.

D. A. Brain, J. H. Engel, R. J. Lillis, R. P. Lin, and D. L. Mitchell, Space Sciences Laboratory, University of California, Berkeley, CA 94720, USA. (rlillis@ssl.berkeley.edu) 